\title{
First-principles study of configurational disorder in B4C using a superatom-special quasirandom structure method
}

\author{
Annop Ektarawong, Sergey Simak, Lars Hultman, Jens Birch and Björn Alling
}

\section{Linköping University Post Print}

\section{Tweet}

N.B.: When citing this work, cite the original article.

Original Publication:

Annop Ektarawong, Sergey Simak, Lars Hultman, Jens Birch and Björn Alling, Firstprinciples study of configurational disorder in B4C using a superatom-special quasirandom structure method, 2014, Physical Review B. Condensed Matter and Materials Physics, (90), 2, 024204.

http://dx.doi.org/10.1103/PhysRevB.90.024204

Copyright: American Physical Society http://www.aps.org/

Postprint available at: Linköping University Electronic Press http://urn.kb.se/resolve?urn=urn:nbn:se:liu:diva-109591 


\title{
First-principles study of configurational disorder in $\mathrm{B}_{4} \mathrm{C}$ using a superatom-special quasirandom structure method
}

\author{
A. Ektarawong, ${ }^{1,}{ }^{*}$ S. I. Simak, ${ }^{2}$ L. Hultman, ${ }^{1}$ J. Birch, ${ }^{1}$ and B. Alling ${ }^{1}$ \\ ${ }^{1}$ Thin Film Physics Division, Department of Physics, Chemistry and Biology (IFM), Linköping University, SE-581 83 Linköping, Sweden \\ ${ }^{2}$ Theoretical Physics Division, Department of Physics, Chemistry and Biology (IFM), Linköping University, SE-581 83 Linköping, Sweden
}

(Received 27 January 2014; revised manuscript received 3 July 2014; published 22 July 2014)

\begin{abstract}
Configurationally disordered crystalline boron carbide, with the composition $\mathrm{B}_{4} \mathrm{C}$, is studied using firstprinciples calculations. We investigate both dilute and high concentrations of carbon-boron substitutional defects. For the latter purpose, we suggest a superatom's picture of the complex structure and combine it with a special quasirandom structure approach for disorder. In this way, we model a random distribution of high concentrations of the identified low-energy defects: (1) bipolar defects and (2) rotation of icosahedral carbon among the three polar-up sites. Additionally, the substitutional disorder of the icosahedral carbon at all six polar sites, as previously discussed in the literature, is also considered. Two configurational phase transitions from the ordered to the disordered configurations are predicted to take place upon an increase in temperature using a mean-field approximation for the entropy. The first transition, at $870 \mathrm{~K}$, induces substitutional disorder of the icosahedral carbon atoms among the three polar-up sites; meanwhile the second transition, at $2325 \mathrm{~K}$, reveals the random substitution of the icosahedral carbon atoms at all six polar sites coexisting with bipolar defects. Already the first transition removes the monoclinic distortion existing in the ordered ground-state configuration and restore the rhombohedral system $(R 3 m)$. The restoration of inversion symmetry yielding the full rhombohedral symmetry $(R \overline{3} m)$ on average, corresponding to what is reported in the literature, is achieved after the second transition. Investigating the effects of high pressure on the configurational stability of the disordered $\mathrm{B}_{4} \mathrm{C}$ phases reveals a tendency to stabilize the ordered ground-state configuration as the configurationally ordering/disordering transition temperature increases with pressure exerted on $\mathrm{B}_{4} \mathrm{C}$. The electronic density of states, obtained from the disordered phases, indicates a sensitivity of the band gap to the degree of configurational disorder in $\mathrm{B}_{4} \mathrm{C}$.
\end{abstract}

DOI: 10.1103/PhysRevB.90.024204

PACS number(s): 64.60.Cn, 64.60.De, 81.30.Hd, 61.50.Ks

\section{INTRODUCTION}

Boron carbide is a class of materials considered for several important applications. The high cross section for thermal neutron reaction of the isotope ${ }^{10} \mathrm{~B}$ makes boron carbide relevant as a new generation of neutron detectors $[1,2]$, possibly replacing the present dominating ${ }^{3} \mathrm{He}$-based technologies suffering from the ${ }^{3} \mathrm{He}$ crisis. Even though only ${ }^{10} \mathrm{~B}$ gives a contribution to detecting neutrons, $\mathrm{B}_{4} \mathrm{C}$ is still considered the most suitable candidate, rather than pure ${ }^{10} \mathrm{~B}$, due to its excellent properties in terms of stability and nontoxicity [3]. In the past few years, prototypes of $\mathrm{B}_{4} \mathrm{C}$-based neutron detectors in the form of multilayer thin solid films have been proposed [1,2]. $\mathrm{B}_{4} \mathrm{C}$ is also used as a shielding material in nuclear reactors due to its ability to absorb neutrons and its unusual ability to heal itself from radiation damage [4-6]. Moreover, $\mathrm{B}_{4} \mathrm{C}$ processes several outstanding properties such as a high hardness, low density, high melting point, low wear coefficient, and high chemical stability. Such properties make it useful as, for example, a lightweight armor, a wear-resistant material, a cutting tool material [7], and a candidate material for high-temperature electronic [8] and thermoelectric [9] devices.

To improve the performance of $\mathrm{B}_{4} \mathrm{C}$ materials in such applications, a detailed understanding of the structure on the atomic level is needed. The structures of $\mathrm{B}_{4} \mathrm{C}$ and related compositions of boron carbide, e.g., $\mathrm{B}_{13} \mathrm{C}_{2}$, have been studied both experimentally and theoretically [10-18] but the substitutional disorder of the carbon atoms has not been

\footnotetext{
*anekt@ifm.liu.se
}

sufficiently investigated. Revealed by diffraction measurements [19-22], $\mathrm{B}_{4} \mathrm{C}$ has a rhombohedral symmetry with the suggested space group $R \overline{3} m$, No. 166 in the International Tables for Crystallography. There are 15 atoms in the rhombohedron unit cell, i.e., 12-atom icosahedra located at vertices of the rhombohedron with a 3-atom linear chain aligned along the [111] direction in the center of the rhombohedron (see Fig. 1). Each icosahedron is formed by two types of crystallographic sites, i.e., six sites for each type, namely, polar and equatorial sites. Atoms in the icosahedron sitting on polar sites form intericosahedral bonds to polar atoms in the neighboring icosahedra. On the other hand, atoms sitting on equatorial sites are linked to the three-atom chains. An arrangement of boron and carbon atoms in the chain is specified, by $\mathrm{X}$-ray diffraction technique [23,24], to be C-B-C. The third carbon atom, as a consequence, must be substituted in the icosahedron. Experimentally identifying the atomic position of the carbon atom in the icosahedron is a formidable task owing to the very close atomic form factors between these two light neighboring elements. First-principles calculations of the energetics of $\mathrm{B}_{4} \mathrm{C}$ has been done by Bylander et al. [10]. They suggested that the substitution of the third carbon atom is at the polar site in the unit cell. The configuration, often denoted $\left(\mathrm{B}_{11} \mathrm{C}^{p}\right)+(\mathrm{C}-\mathrm{B}-\mathrm{C})$, yields the lowest total energy. The same conclusion was later confirmed by theoretical inspection of vibrational properties [12] and by a first-principles analysis of NMR spectra [13]. Such a substitution of the carbon atom also reduces the symmetry, by a small distortion, from rhombohedral to base-centered monoclinic (space group $\mathrm{Cm}$; No. 8). However, an experimental observation of that monoclinic $\mathrm{B}_{4} \mathrm{C}$ phase has never been reported. The reason 


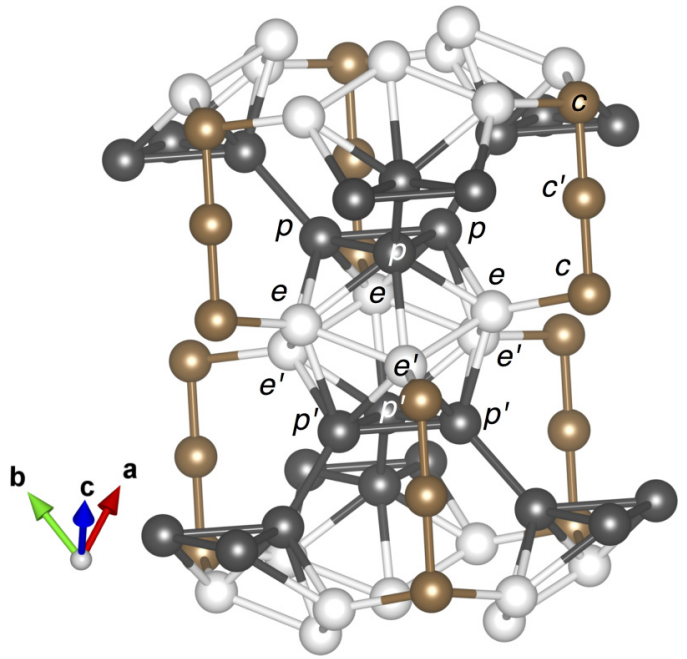

FIG. 1. (Color online) Icosahedral structure of boron carbide. Black, white, and brown spheres represent polar (up, $p$; down, $p^{\prime}$ ), equatorial (up, $e$; down, $e^{\prime}$ ), and chain (end, $c$; center, $c^{\prime}$ ) sites, repectively.

has been suggested [12] to be due to configurational disorder, in particular, a disordered substitution of carbon atoms among all six polar sites with equal concentrations. Such disorder should restore the higher rhombohedral symmetry found in experiments. Apart from the substitutional disorder of the carbon atom among the polar sites in the icosahedra, substitution of the polar carbon from one icosahedron to a neighboring icosahedron forming $\left(\mathrm{B}_{10} \mathrm{C}_{2}^{p}\right)+\left(\mathrm{B}_{12}\right)$, so-called bipolar defects (BDs), are demonstrated to exist in the structure [13].

Disorder in boron carbide has been studied both experimentally [25-27] and theoretically [14,28-31]. Moreover, thin films of $\mathrm{B}_{4} \mathrm{C}$, which are more or less understoichiometric, are

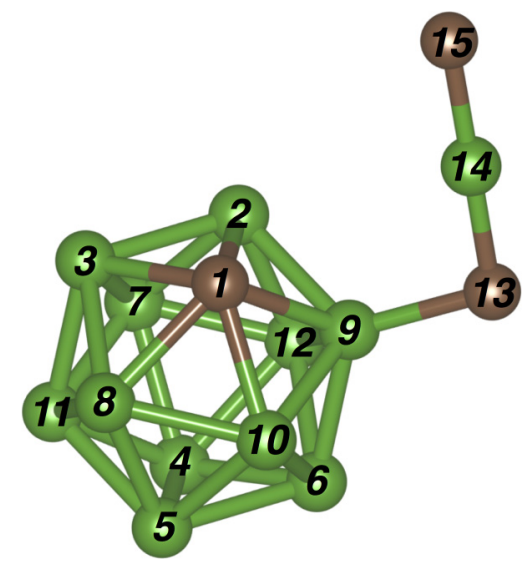

FIG. 2. (Color online) A 12-atom icosahedron and a 3-atom chain made of boron (green) and carbon (brown) atoms. A set of numbers $(1,2,3)$ denotes the polar-up sites. Similarly, the set $(4$, $5,6)$ denotes the polar-down sites. The equatorial-up $(7,8,9)$, equatorial-down $(10,11,12)$, and chain $(13,14,15)$ sites are included, corresponding to the numbers in parentheses. A dilute defect, namely, "a rotation of the icosahedral carbon atom among the polar-up sites," occurs when a carbon atom at position 1 swaps its position with a boron atom at position 2 or 3 .

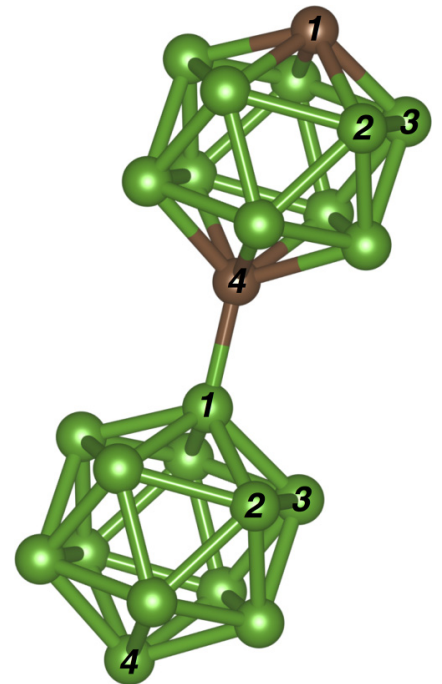

FIG. 3. (Color online) A bipolar defect involved with two icosahedra, i.e., $\left(\mathrm{B}_{11} \mathrm{C}_{2}^{p}\right)+\left(\mathrm{B}_{12}\right)$. Numbers and colors indicate the same positions and types of atoms, respectively, as described in the caption to Fig. 2. Three-atom chains are not shown.

amorphous when grown at low temperatures, i.e., topologically disordered [32]. Despite the evidence that existing samples of $\mathrm{B}_{4} \mathrm{C}$ are configurationally disordered, no theoretical method has been suggested to directly calculate the properties of such disordered structures. Therefore, we present in this work a theoretical technique that provides a path to investigate configurationally disordered $\mathrm{B}_{4} \mathrm{C}$, based on first-principles calculations. We start with a detailed study of dilute defects, with focus on the site displacement of the icosahedral carbon atom. Various types of dilute defects are introduced into sufficiently big supercells of ideally ordered $\mathrm{B}_{4} \mathrm{C}$. As seen by determining defect formation energies, we single out which kinds of defects are most likely to appear in the structure during synthesis. We demonstrate that a rotation of the icosahedral carbon atom among the polar-up sites (positions 1, 2, and 3 in Fig. 2) and a BD (see Fig. 3) are the most likely ones. Note that in this work, for all cases the ratio of the boron to the carbon concentrations retains the $4: 1$ stoichiometry (20\% atomic carbon concentration). Also, all of the configurations considered are electrically neutral. In the next step, to study substitutionally disordered configurations of $\mathrm{B}_{4} \mathrm{C}$, we suggest a method to distribute the identified most important defect types with high concentrations. The method, superatom special quasirandom structure (SA-SQS), is based on a combination of the special quasirandom structure (SQS) approach, originally suggested by Zunger et al. [33], and a concept of superatoms of different icosahedral structures, which are identified through dilute defect calculations. This allows us to investigate theoretically, one step closer to the real situation, the properties, such as symmetry and electronic density of states (DOS), of disordered $\mathrm{B}_{4} \mathrm{C}$ phases and to model order-disorder transitions.

\section{COMPUTATIONAL DETAILS}

All calculations were done based on density functional theory. The projector augmented wave (PAW) method, as 
implemented in the Vienna ab initio simulation package (VASP) [34,35], was utilized to calculate the total energy of the boron carbide systems. The Perdew-Becke-Ernzerhof (PBE96) generalized gradient approximation (GGA) [36] was chosen for the exchange-correlation functional. The monoclinic-distorted $\mathrm{B}_{4} \mathrm{C}$, based on a 15-atom unit cell $\left[\left(\mathrm{B}_{11} \mathrm{C}^{p}\right)+(\mathrm{C}-\mathrm{B}-\mathrm{C})\right]$, was used as the starting point for further calculations. The atomic coordinates and the cell shape were fully relaxed in each structural case. The equilibrium volume was then obtained from a curve plotted for the total energies versus the fixed volumes. Energy cutoffs of $400 \mathrm{eV}$ were set for plane-wave calculations. The total energy was converged within an accuracy of $1 \mathrm{meV} /$ atom, with respect to both the energy cutoff and the number of $\mathbf{k}$ points used for integration over the Brillouin zone. A $5 \times 5 \times 5$ Monkhorst-Pack k-point mesh [37] was used for the 15-atom unit cell, and for supercell sizes up to only $2 \times 2 \times 2$ (120 atoms), while supercell sizes larger than $2 \times 2 \times 2$, i.e., $3 \times 3 \times 3$ (405 atoms) and $4 \times 3 \times$ 3 (540 atoms), were sampled with a $3 \times 3 \times 3$ MonkhorstPack k-point mesh. For electronic DOS calculations, the tetrahedron method for Brillouin-zone integrations suggested by Blöchl [38] was used with a $9 \times 9 \times 9$ Monkhorst-Pack k-point mesh for the 15-atom unit cell and for supercell sizes up to $2 \times 2 \times 2$ and with a $5 \times 5 \times 5$ Monkhorst-Pack k-point mesh for supercell sizes larger than $2 \times 2 \times 2$. Additionally, the modified Becke-Johnson (MBJ) exchange potential $[39,40]$ in combination with the GGA-PBE correlation was applied to DOS calculations for all configurations with a $5 \times 5 \times 5$ Monkhorst-Pack k-point mesh. For benchmarking, the hybrid functional HSE06 [41,42] was applied to DOS calculations for the 15 -atom ordered unit cell.

To study the effects on the $\mathrm{B}_{4} \mathrm{C}$ system when different kinds of dilute defects were introduced, supercell sizes up to $2 \times 2 \times 2$ unit cells (120 atoms) were mainly used in the calculations. This supercell size ensures that the interactions between the defects are small and the defect formation energies were obtained with a size-dependent uncertainty of less than $40 \mathrm{meV}$, as judged from comparison to $3 \times 3 \times 3$ supercell (405-atom) calculations. As mentioned in the previous section, for all cases the atomic carbon concentration was kept fixed at $20 \%$; each type of a dilute defect was thus created with respect to the site displacement of an icosahedral carbon atom in one specific icosahedron in the supercell. During calculations, only the atomic coordinates were allowed to relax and the input volume was fixed based on the equilibrium one. The defect formation energy ( $\left.\Delta E_{\text {defect }}\right)$ was directly calculated by the equation

$$
\Delta E_{\text {defect }}=E_{\text {defect }}-E_{\left[\mathrm{B}_{11} \mathrm{C}^{p}\right]+[\mathrm{C}-\mathrm{B}-\mathrm{C}]},
$$

where $E_{\text {defect }}$ and $E_{\left[\mathrm{B}_{11} \mathrm{C}^{p}\right]+[\mathrm{C}-\mathrm{B}-\mathrm{C}]}$ are defined as the total energy of the system with and without a defect, respectively. Fourteen types, in total, of dilute defects were considered, as listed in Table I, including their formation energies. Regarding the stability under zero-pressure and zero-temperature conditions for all configurations of $\mathrm{B}_{4} \mathrm{C}$, the formation energy with respect to decomposition into elemental phases, i.e., in this work, $\alpha$-boron and diamond, was also considered. Visualizations of various configurations were obtained with the VESTA package [43].
TABLE I. Fourteen studied types of dilute defects in $2 \times 2 \times 2$ supercells and their formation energies with respect to the $\mathrm{B}_{11} \mathrm{C}^{p(1)}+$ (C-B-C) structure, which is used as a reference. Superscripts $p$ and $e$ denote polar and the equatorial sites in the icosahedron, respectively. The superscript of the number in parentheses corresponds to the position in the icosahedron as indicated in Fig. 2. For the three special cases of distorted bipolar defects (nos. 1-3), see Fig. 5.

\begin{tabular}{|c|c|}
\hline Defective structure & Formation energy $(\mathrm{eV})$ \\
\hline \multicolumn{2}{|l|}{ Nondefective } \\
\hline $\mathrm{B}_{11} \mathrm{C}^{p(1)}+(\mathrm{C}-\mathrm{B}-\mathrm{C})$ & 0 \\
\hline \multicolumn{2}{|l|}{ Bipolar defect } \\
\hline \multirow{4}{*}{$\mathrm{B}_{10} \mathrm{C}_{2}^{p(1,4)}+\mathrm{B}_{12}+(\mathrm{C}-\mathrm{B}-\mathrm{C})_{2}$} & 0.229 \\
\hline & $0.262^{\mathrm{a}}$ \\
\hline & $\sim 0.23^{\mathrm{b}}$ \\
\hline & $0.477^{\mathrm{c}}$ \\
\hline \multicolumn{2}{|l|}{ Polar sites } \\
\hline \multirow[t]{3}{*}{$\mathrm{B}_{11} \mathrm{C}^{p(2,3)}+(\mathrm{C}-\mathrm{B}-\mathrm{C})$} & 0.232 \\
\hline & $0.248^{\mathrm{a}}$ \\
\hline & $0.135^{\mathrm{c}}$ \\
\hline $\mathrm{B}_{11} \mathrm{C}^{p(4)}+(\mathrm{C}-\mathrm{B}-\mathrm{C})$ & 1.217 \\
\hline $\mathrm{B}_{11} \mathrm{C}^{p(5,6)}+(\mathrm{C}-\mathrm{B}-\mathrm{C})$ & 0.464 \\
\hline \multicolumn{2}{|l|}{ Equatorial sites } \\
\hline $\mathrm{B}_{11} \mathrm{C}^{e(7)}+(\mathrm{C}-\mathrm{B}-\mathrm{C})$ & 0.674 \\
\hline $\mathrm{B}_{11} \mathrm{C}^{e(8,9)}+(\mathrm{C}-\mathrm{B}-\mathrm{C})$ & 0.714 \\
\hline $\mathrm{B}_{11} \mathrm{C}^{e(10)}+(\mathrm{C}-\mathrm{B}-\mathrm{C})$ & 0.598 \\
\hline \multirow[t]{2}{*}{$\mathrm{B}_{11} \mathrm{C}^{e(11,12)}+(\mathrm{C}-\mathrm{B}-\mathrm{C})$} & 0.793 \\
\hline & $\sim 0.74^{\mathrm{b}}$ \\
\hline \multicolumn{2}{|l|}{ A-chain-centered site } \\
\hline \multirow[t]{2}{*}{$\mathrm{B}_{12}+(\mathrm{C}-\mathrm{C}-\mathrm{C})$} & 1.445 \\
\hline & $\sim 1.69^{\mathrm{b}}$ \\
\hline \multicolumn{2}{|l|}{ Disordered chain } \\
\hline $\mathrm{B}_{11} \mathrm{C}^{p(1)}+(\mathrm{B}-\mathrm{C}-\mathrm{C})$ & 2.521 \\
\hline $\mathrm{B}_{11} \mathrm{C}^{p(1)}+(\mathrm{C}-\mathrm{C}-\mathrm{B})$ & 2.598 \\
\hline \multicolumn{2}{|l|}{ Distorted bipolar defect } \\
\hline No. 1 & 0.628 \\
\hline No. 2 & 1.066 \\
\hline No. 3 & 1.152 \\
\hline
\end{tabular}

${ }^{\mathrm{a}}$ From this work; $3 \times 3 \times 3$ supercell.

${ }^{\mathrm{b}}$ From Ref. [30]; $2 \times 2 \times 2$ supercell (LDA-PP).

${ }^{\mathrm{c}}$ From Ref. [31]; $3 \times 1 \times 1$ supercell (GGA-PW91).

\section{SUPERATOM-SPECIAL QUASIRANDOM STRUCTURE TECHNIQUE}

Modelling of configurational disorder beyond dilute defects in complex crystal structures, such as $\mathrm{B}_{4} \mathrm{C}$, using the complete mathematical apparatus of Sanchez, Ducastelle, and Gratias [44] is often impractical. Furthermore, it is not necessary to take into account all kinds of defects in disorder models, and many of them might be neglected due to high total energies. Instead, in such cases, one can try to reveal the important physics by focusing on the modeling of low-energy disordered patterns. Here, we suggest that configurationally disordered $\mathrm{B}_{4} \mathrm{C}$ can be viewed as a disordered distribution of different superatoms, each defined based on knowledge of low-energy dilute defects. We suggest the SA-SQS technique to model this situation in $\mathrm{B}_{4} \mathrm{C}$. In this approach, the configuration of the superatoms is modeling a random alloy pattern according to the SQS approach. In our case, a 12-atom icosahedron together 


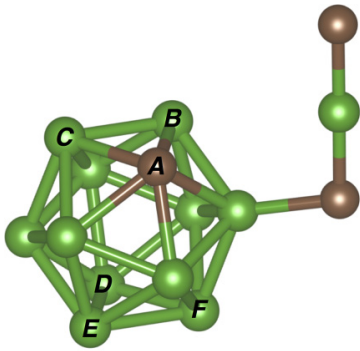

(a)

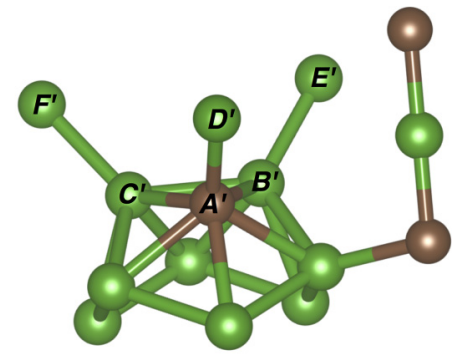

(b)
FIG. 4. (Color online) Two kinds of superatom bases for constructing disordered configurations in $\mathrm{B}_{4} \mathrm{C}$ : (a) Basis-1 and (b) Basis-2. Green and brown spheres represent boron and carbon atoms, respectively. The types of superatoms are distinguished by the respective positions of the icosahedral carbon atom. Letters $A$ to $F\left(A^{\prime}\right.$ to $\left.F^{\prime}\right)$ denote the type of superatom and also the position of the icosahedral carbon atom for Basis-1 (Basis-2).

with a 3-atom chain was treated as a superatom unit. The first intuitive way to define this superatom basis is to identify the 15 atoms of the $\mathrm{B}_{4} \mathrm{C}$ unit cell as the superatom basis [see Fig. 4 (a)]. However, to allow for the description of the two types of dilute defects that we find to have the distinctly lowest formation energy, i.e., the $\mathrm{BD}$ and the polar-up carbon rotation, a second way of defining the superatom basis was introduced [see Fig. 4(b)]. It replaces, within the basis, the polar-down sites from a single icosahedron with the corresponding polardown sites of neighboring icosahedra with bonds to the original polar-up sites. A configurational disorder, based on either the $\mathrm{BD}$ or the carbon rotation among the polar sites, can then be created.

For the creation of disordered configurations of superatoms, we used equal concentrations of each included superatom type and the SQS technique to randomly distribute them with the aim of getting the Warren-Cowley short-range-order parameters $\left(\alpha_{i} ; i=1-4\right)$ for the first four coordination shells to be as close to 0 as possible. As mentioned in the previous paragraph, we introduced two kinds of superatom bases. Both bases allow for six different superatom types, with respect to the position of the icosahedral carbon atom at any of the six polar sites (see Fig. 4). In the present work, six different disordered configurations, constructed using the SA-SQS technique, were considered: We include configurations with two types of superatoms (modeling BDs), three types of superatoms (modeling rotation of carbon atoms on the three polar-up sites), four types of superatoms (modeling rotation on polar-up sites in combination with one type of $\mathrm{BD}$ ), and six types of superatoms (modeling rotation of carbon atoms on all six polar sites) The structures used in the calculations were based on $3 \times 3 \times 3$ (405-atom) and $4 \times 3 \times 3$ (540-atom) supercells. For each disordered configuration, the atomic coordinates and cell shape were optimized in a series of different fixed-volume calculations.

The Gibbs free energy under zero-pressure conditions were calculated for all configurations, where the configurational entropy was determined within the mean-field approximation. The transition temperatures were then estimated from a plot of the free energy as a function of the temperature $T$. The equation for the free energy calculation is

$$
G^{\gamma}=E^{\gamma}-T S^{\gamma}
$$

where $G^{\gamma}, E^{\gamma}$, and $S^{\gamma}$ stand for the Gibbs free energy, the total energy, and the configurational entropy of the system with configuration $\gamma$. The entropy $S$ was obtained within the thermodynamics limit, i.e., the number of particles approaches infinity, using Stirling's approximation of the binomial distribution. A general expression for the configurational entropy $S$ in this so-called mean-field approximation is

$$
S=-k_{B} N \sum_{i=1}^{n} x_{i} \ln \left(x_{i}\right),
$$

where $N$ and $n$ are defined as the number of superatom sites in the supercell and the number of superatom types included in the supercell, respectively. $x_{i}$ refers to the concentration of type $i$ superatoms. If the concentration of each type of superatom is assigned to be equal, the entropy per superatom site is thus reduced to

$$
S=k_{B} \ln (n),
$$

where $n$ is the number of types of superatoms defined for each configuration.

To investigate the effects of pressure on the configurational stability of $\mathrm{B}_{4} \mathrm{C}$, the total energies at different fixed volumes of some selected configurations were fitted to the third-order Birch-Murnaghan equation of state $[45,46]$. The Gibbs free energy of a zero-pressure condition according to Eq. (2) is, therefore, modified and given by

$$
G^{\gamma}=E^{\gamma}+P V^{\gamma}-T S^{\gamma},
$$

where $P$ and $V^{\gamma}$ are defined as the applied pressure and the equilibrium volume of the system of configuration $\gamma$ corresponding to the pressure $P$, respectively.

\section{RESULTS AND DISCUSSION}

\section{A. Dilute defects}

Based on our calculations, we find that the different sites of the unit cell of ideally ordered $\mathrm{B}_{4} \mathrm{C}$ are nonequivalent in defect formation aspect to a higher degree than has been previously realized. The calculated defect formation energies per defect of dilute configurational defects according to Eq. (1) are listed in Table I. Most of them are calculated in a supercell of 120 atoms $(2 \times 2 \times 2$ repetition of the unit cell). All defects increase the energy with respect to ideally ordered $\mathrm{B}_{4} \mathrm{C}$. However, these defective structures are still very stable with respect to $\alpha$-boron and diamond. This is not surprising based on their dilute character and the high stability of the perfect $\mathrm{B}_{4} \mathrm{C}$ phase. Note that the atomic position of the carbon atom in the icosahedron is denoted by $p$ or $e$, depending on its position at the polar or equatorial sites, respectively, and the numbers refer to the positions according to the notation in Fig. 2. For example, the notation $p(1)$ corresponds to an icosahedron with a carbon atom positioned according to Fig. 2, which is the case for all icosahedra if the ground-state (GS) configuration, $\mathrm{B}_{11} \mathrm{C}^{p(1)}+(\mathrm{C}-\mathrm{B}-\mathrm{C})$, is considered. The defect with the lowest energy with respect to the GS configuration is the $\mathrm{BD}\left(\Delta E_{\text {defect }}=0.229 \mathrm{eV}\right)$, illustrated in Fig. 3. As reported 
in Table I, our calculated energy is in good agreement with the result calculated by Raucoules et al. [30], where the supercell sizes used for the calculations are the same $2 \times 2 \times 2$. On the other hand, the BD formation energy provided by Widom and Huhn [31] is almost two times higher than our result. We suggest that the discrepancy in the formation energy involves the difference in supercell sizes. Since their calculations were done in a $3 \times 1 \times 1$ supercell, it is questionable that the supercell was large enough to avoid uncontrolled defect-defect interaction effects. To verify the accuracy of our result, we perform a calculation of the BD formation energy in a $3 \times$ $3 \times 3$ supercell. The result shows that the formation energy in the $3 \times 3 \times 3$ supercell differs by only $0.03 \mathrm{eV} /$ defect from that of the $2 \times 2 \times 2$ case. Furthermore, these BDs have been reported to be presented in the structure, as native defects formed during the synthesis process [30].

Another type of defect, coming after the bipolar one, is a rotation of the icosahedral carbon atom among the polar-up sites $\left(\Delta E_{\text {defect }}=0.232 \mathrm{eV}\right)$, i.e., the substitution of the carbon atom at the $p(2$, or 3$)$ position instead of the $p(1)$ position. Note that moving the icosahedral carbon atom to either $p(2)$ or $p(3)$ is equivalent and they thus give the same formation energies. This type of defect has a formation energy only slightly higher than that of the BD. However, when even larger, $3 \times 3 \times 3$ supercells are used, the formation energies of bipolar and polar-up carbon rotation defects are actually changing order even though the defect formation energies change by about $0.04 \mathrm{eV} /$ defect, meaning that these two types of defects are practically equal in energy. Vast et al. [14] suggested that it is the random substitution of carbon atoms among all six polar sites of the icosahedra that results in the rhombohedral symmetry $(R \overline{3} m)$ of $\mathrm{B}_{4} \mathrm{C}$ observed in experiments. A similar conclusion was made by Widom and Huhn [31]. Based on our calculations, the energy it costs to swap a carbon atom to polar-down sites is higher than the swapping of a carbon atom only among the polar-up sites. That is, the energy to flip an icosahedral carbon atom to the $p(5$ or 6$)$ and $p(4)$ positions becomes approximately twice $(0.46 \mathrm{eV})$ and six times $(1.21 \mathrm{eV})$, respectively that of the polar-up carbon rotation. The latter case can be thought of as flipping the icosahedron upside down, thus resulting in an intericosahedral $\mathrm{C}-\mathrm{C}$ bond. Due to its high formation energy, the $\mathrm{C}-\mathrm{C}$ bond is considered an unfavorable type of bonding for the $\mathrm{B}_{4} \mathrm{C}$ system as demonstrated also by the case of $\left[\left(\mathrm{B}_{12}\right)+(\mathrm{C}-\mathrm{C}-\mathrm{C})\right]$, i.e., a chain consisting only of carbon atoms, with a formation energy of $1.44 \mathrm{eV}$. Hence, we expect that icosahedral carbon atoms are more favorable for substitution of polar sites on only one side rather than on both the up and the down sides. We return to the consequence of polar carbon rotation-based disordered configurations in Sec. IV B. Note that apart from these two types of dilute defects, i.e., the BD and polar-up carbon rotation, the remainder provides significantly higher formation energies and thus they are expected to be present in the structure at much lower concentrations.

The nonequivalence of sites also goes for the equatorial and the chain sites. Obviously, the three equatorial-up sites $e(7,8,9)$ are not equivalent to the equatorial-down ones $e(10,11,12)$, similarly to the case of the polar sites. More importantly, one site of both the equatorial-up and the equatorial-down sites is different from the other two sites as revealed by their defect formation energies. The two equatorial-up sites $e(8,9)$ are different from the other one $e(7)$. Also, the two equatorial-down sites $e(11,12)$ are not equivalent to the other equatorial-down site $e(10)$. Especially for the equatorial-down sites, the difference in the formation energy between these nonequivalent sites is as much as $0.2 \mathrm{eV}$, which is comparable to the formation energy of a BD as well as of rotation of the carbon atom among polar-up sites. In the case of chain sites, swapping between boron and carbon atoms sitting on the chain sites in the opposite direction, i.e., $\left[\left(\mathrm{B}_{11} \mathrm{C}^{p}\right)+(\mathrm{B}-\mathrm{C}-\mathrm{C})\right]$ and $\left[\left(\mathrm{B}_{11} \mathrm{C}^{p}\right)+(\mathrm{C}-\mathrm{C}-\mathrm{B})\right]$, also results in different formation energies, indicating nonequivalence at the chain sites. To our knowledge, this degree of nonequivalence of the sites in ideally ordered $\mathrm{B}_{4} \mathrm{C}$, both in the icosahedron and in the chain, have not been pointed out previously. In summary, based on the energetics of dilute defects one can assume that the BD and polar-up rotation of icosahedral carbon atoms can be expected to be present in $\mathrm{B}_{4} \mathrm{C}$ at high concentrations under equilibrium high-temperature conditions. Other types of defects that are at least twice as high in energy can still be present as dilute defects or as consequences of out-of-equilibrium synthesis with, e.g., insufficient diffusion during sample preparation.

\section{B. Disorder configurations of $\mathrm{B}_{\mathbf{4}} \mathrm{C}$}

According to what was indicated in the previous subsection, we conclude that two types of defects, (1) the BD and (2) a rotation of the icosahedral carbon atom among the polar-up sites, are the most probable defects to be present in a structure with high concentrations at equilibrium. The disordered configurations were, therefore, constructed mainly based on these two types of dilute defects. However, as the disorder of carbon atoms at all six polar sites has been discussed in the literature, this type of disorder was also considered. As described in Sec. III, we suggest two kinds of superatom bases for constructing the disordered configurations, as shown in Fig. 4. Clearly for the Basis-1 superatom, a whole icosahedral structure with a three-atom chain is defined as one superatom. Using this basis, a disordered configuration, in which the icosahedral carbon atom in each icosahedron can substitute any of the six polar sites or any of only the three polar-up sites, can be obtained. However, Basis-1 alone is not enough to describe BDs. This is because for a BD not only the coexistence of $\mathrm{B}_{12}$ and $\mathrm{B}_{10} \mathrm{C}_{2}$ icosahedra, but also their particular arrangements need to be taken into consideration, as can be seen in the energy difference between the BD and the distorted BDs (see Fig. 5). To handle such difficulties, the use of Basis-2 superatoms becomes important.

In the present work, seven different disordered configurations are considered. Among these seven configurations, six were constructed using our SA-SQS technique (see Fig. 6 for the SA-SQS describing polar-up rotation), while the seventh configuration was created using a random number generator (RNG) to distribute all 27 icosahedral carbon atoms among the 162 polar sites in supercell in an uncorrelated manner. The configurational details of seven different disordered configurations are listed in Table II. The short-range-order parameters $\alpha_{i}$ are 0 up to the fourth shell for the BD-based disordered configuration. For the 3PU configuration, where the icosahedral carbon atoms are allowed to substitute only 


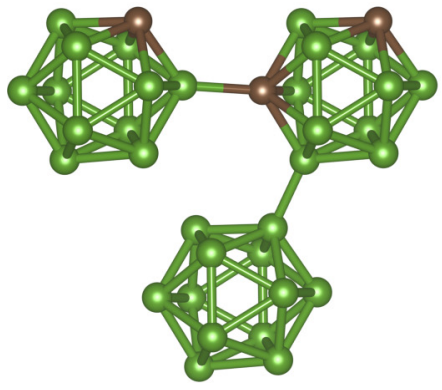

(a)

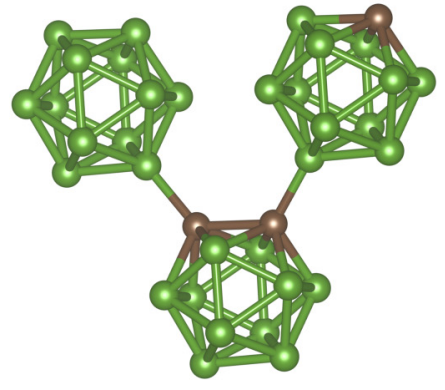

(b)

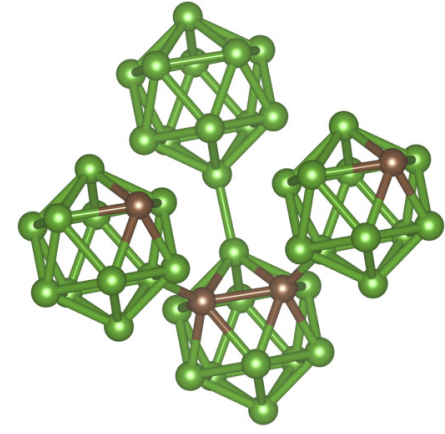

(c)

FIG. 5. (Color online) Three cases of distorted bipolar defects: (a) no. 1, (b) no. 2, and (c) no. 3. Green and brown spheres represent boron and carbon atoms, respectively. Three-atom chains are not shown.

the three polar-up sites, the short-range-order parameters are 0 for the first and second shells. Small nonzero SRO values are present for the other multicomponent cases, and supercell sizes larger than $4 \times 3 \times 3$ would be required to obtain an ideal random alloy model. Unfortunately, due to the 15 atoms per superatom basis, even larger supercells would not be computationally affordable in the present work. As a consequence, we have limited the supercell size to $4 \times 3 \times 3$ superatoms, corresponding to 540 individual atoms. The SA-SQS structure files in VASP format of the fully relaxed 3PU-, 6P-, and (BD + 6P)-disordered configurations are included as Supplementary Material [47].

In Table II the energy with respect to the configurational GS, the type of superatoms included, the supercell size, and the lattice parameters for each case are reported. The disordered configuration based on BDs is found to be $0.066 \mathrm{eV}$ per superatom higher in energy than the configurational GS. The rotation of carbon atoms among polar-up sites (3PU) is $0.083 \mathrm{eV}$ per superatom higher than the GS. Then follow, in order of increasing energy, the combination of BDs with polarup rotation in Basis-2 and three polar-up rotation together with one of the polar-down sites in Basis-1 and the two cases with six types of superatoms with carbon disordered among all polar sites in the two respective bases. Highest in energy is the configuration created with an RNG distribution of polar site carbon. Again, the phase stabilities against phase

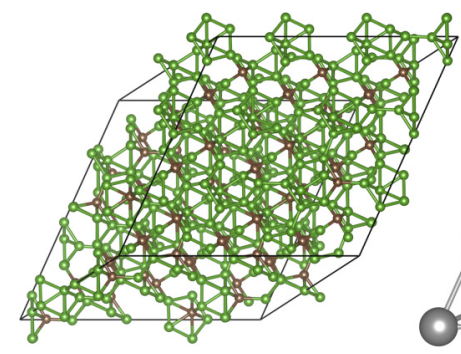

(a)

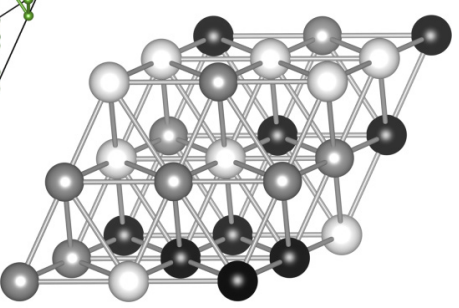

(b)
FIG. 6. (Color online) $3 \times 3 \times 3$ supercells of the (3PU)disordered configuration in $\mathrm{B}_{4} \mathrm{C}$ represented in (a) a normal-atom picture and (b) a superatom picture. (a) Green and brown spheres represent boron and carbon atoms, respectively. (b) Black, white, and gray spheres represent superatom types $A, B$, and $c$ for Basis-1, respectively. decomposition into $\alpha$-boron and diamond for all disordered configurations listed in Table II are considered. Calculating the formation energy indicates that they are stable against phase decomposition, where their formation energies fall into the range between -0.130 and $-0.093 \mathrm{eV} /$ atom for the most stable (GS) configuration and least stable (RNG) configuration, respectively.

By comparing the lattice parameters between the disordered configurations and the GS, effects of disorder on the symmetry of the system can be found. The substitutional disorder of carbon atoms among all six polar sites in the $6 \mathrm{P}$ configuration fully restores the rhombohedral symmetry $(R \overline{3} m)$ on average. This is in line with previous suggestions [14,31]. However, here we find that the substitutional disorder of carbon atoms among the three polar-up sites only is sufficient to restore the rhombohedral system. It, however, lacks inversion symmetry. This loss of the inversion symmetry in the 3PU configuration thus yields the space group $R 3 m$, no. 160 . In addition to a rotation of the carbon atom among three polar-up sites, we have also considered the 3PU+1PD configuration, which allows the icosahedral carbon atoms to substitute for the three polar-up and one of the polar-down sites. This configuration is less favorable compared to the 3PU configuration because (1) the energy required to move a carbon atom to the polar-down sites is rather high, as indicated in Sec. II, and (2) there is the possibility of intericosahedral $\mathrm{C}-\mathrm{C}$ bond formation. The degree of monoclinic distortion, in the case of the 3PU+1PD configuration, is smaller, placing its structure somewhere in between that of the GS and that of the 3PU or 6P configuration. We suggest that the decrease in monoclinic distortion in this case is due to the competitive effects between an equal distribution of the carbon atoms among the three polar-up sites, which is trying to restore rhombohedral symmetry, and a substitution of the carbon atoms at only one of the polar-down sites, which induces the monoclinic distortion. The existence of BDs does not induce any change with respect to the the lattice parameters and the angles. They are all practically the same as of the GS configuration. The monoclinic distortion remains in the $\mathrm{BD}$ configuration.

The $a, b$, and $c$ lattice parameters ( $\alpha, \beta$, and $\gamma$ angles) are in fact not exactly identical even for the 6P, the 3PU, and the $\mathrm{BD}+6 \mathrm{P}$ configurations. However, the magnitude of the difference is small [of the order of $0.001 \AA$ (degree)]. However, from a practical point of view they are the same. 
TABLE II. Configurational details of seven disordered configurations in $\mathrm{B}_{4} \mathrm{C}$. GS-ground-state configuration of $\mathrm{B}_{4} \mathrm{C}$ with a monoclinic distortion, which is a referential configuration; $\mathrm{BD}$ — bipolar defect-based disordered configuration; $x \mathrm{P}, x \mathrm{PU}$, or $x \mathrm{PD}$ — disordered configuration constructed based on substituting carbon atoms at the polar, polar-up, or polar-down sites, respectively, where $x$ is the number of sites that are substituted by carbon atoms; RNG-disordered configuration obtained by randomly distributing all icosahedral carbon atoms among the polar sites in the structure using a random number generator; $\Delta E$-difference in energy per superatom (s.a.) of the disordered configurations with respect to the ground state; $\Delta E^{\text {form }}$ - formation energy with respect to a phase decomposition into $\alpha$-boron and diamond.

\begin{tabular}{|c|c|c|c|c|c|c|c|c|c|c|}
\hline \multirow[b]{2}{*}{ Configuration } & \multirow{2}{*}{$\begin{array}{c}\Delta E \\
(\mathrm{eV} / \text { s.a. })\end{array}$} & \multirow{2}{*}{$\begin{array}{c}\Delta E^{\text {form }} \\
(\mathrm{eV} / \text { atom })\end{array}$} & \multirow{2}{*}{$\begin{array}{l}\text { Type(s) of } \\
\text { superatom }\end{array}$} & \multirow{2}{*}{$\begin{array}{l}\text { Supercell size } \\
\text { (no. of atoms) }\end{array}$} & \multicolumn{3}{|c|}{$\begin{array}{l}\text { Lattice parameter } \\
\text { (§/unit cell) }\end{array}$} & \multicolumn{3}{|c|}{ Angle (deg) } \\
\hline & & & & & $a$ & $b$ & $c$ & $\alpha$ & $\beta$ & $\gamma$ \\
\hline GS & 0 & -0.130 & $A$ & $4 \times 3 \times 3(540)$ & 5.209 & 5.209 & 5.059 & 66.01 & 66.01 & 65.14 \\
\hline $\mathrm{GS}^{\mathrm{a}}$ & - & - & - & $1 \times 1 \times 1(15)$ & 5.143 & 5.143 & 5.010 & 66.39 & 66.39 & 65.57 \\
\hline BD & 0.066 & -0.126 & $A^{\prime}, D^{\prime}$ & $4 \times 3 \times 3(540)$ & 5.209 & 5.209 & 5.060 & 66.01 & 66.00 & 65.14 \\
\hline 3PU & 0.083 & -0.125 & $A, B C$ & $3 \times 3 \times 3(405)$ & 5.159 & 5.159 & 5.159 & 65.73 & 65.73 & 65.73 \\
\hline $3 \mathrm{PU}+1 \mathrm{PD}$ & 0.179 & -0.118 & $A, B, C, D$ & $4 \times 3 \times 3(540)$ & 5.173 & 5.173 & 5.136 & 65.79 & 65.79 & 65.58 \\
\hline $6 \mathrm{P}$ & 0.226 & -0.115 & $\begin{array}{c}A, B, C \\
D, E, F\end{array}$ & $4 \times 3 \times 3(540)$ & 5.161 & 5.161 & 5.161 & 65.72 & 65.72 & 65.72 \\
\hline $\mathrm{BD}+3 \mathrm{PU}$ & 0.163 & -0.119 & $A^{\prime}, B^{\prime}, C^{\prime}, D$ & $4 \times 3 \times 3(540)$ & 5.172 & 5.172 & 5.135 & 65.80 & 65.79 & 65.58 \\
\hline $\mathrm{BD}+6 \mathrm{P}$ & 0.222 & -0.116 & $\begin{array}{l}A^{\prime}, B^{\prime}, C^{\prime} \\
D^{\prime}, E^{\prime}, F^{\prime}\end{array}$ & $4 \times 3 \times 3(540)$ & 5.161 & 5.161 & 5.161 & 65.73 & 65.73 & 65.73 \\
\hline RNG & 0.553 & -0.093 & - & $3 \times 3 \times 3(405)$ & 5.151 & 5.162 & 5.172 & 65.66 & 65.76 & 65.78 \\
\hline Experiment $^{\mathrm{b}}$ & - & - & - & - & 5.163 & 5.163 & 5.163 & 65.73 & 65.73 & 65.73 \\
\hline Experiment $^{\mathrm{c}}$ & - & - & - & - & 5.155 & 5.155 & 5.155 & 65.68 & 65.68 & 65.68 \\
\hline
\end{tabular}

${ }^{\mathrm{a}}$ From Ref. [48]; LDA-PP.

${ }^{\mathrm{b}}$ From Ref. [22]; neutron diffraction.

${ }^{\mathrm{c}}$ From Ref. [23]; x-ray diffraction.

Thus, practically, and exactly in a hypothetical infinite-sized supercell, rhombohedral symmetry $(R \overline{3} m)$ is restored on average for the $6 \mathrm{P}$, and the $\mathrm{BD}+6 \mathrm{P}$ configurations, while the lower symmetry $(R 3 m)$ without the inversion is restored for the $3 \mathrm{PU}$ configuration. The lattice parameters and the angles of those having rhombohedral symmetry (both $R 3 m$ and $R \overline{3} m$ ) are in very good agreement with the experimental result obtained from neutron diffraction [22] as listed in Table II.

The configurational results reveal the monoclinic distortion decreases in the $(\mathrm{BD}+3 \mathrm{PU})$ configuration, which is more or less the same as that $\mathrm{ub}$ the $3 \mathrm{PU}+1 \mathrm{PD}$ configuration. On the other hand, the $\mathrm{BD}+6 \mathrm{P}$ configuration, as mentioned above, has rhombohedral symmetry $(R \overline{3} m)$. The explanation for both the smaller monoclinic distortion in the BD + 3PU configuration and the restoration of the higher symmetry in the $\mathrm{BD}+6 \mathrm{P}$ configuration can be given similarly as in the case of the $3 \mathrm{PU}+1 \mathrm{PD}$ and $6 \mathrm{P}$ configurations, respectively. The RNG configuration is the most configurationally disordered, since the icosahedral carbon atoms are allowed to sit nonspecifically on any of the polar sites in the structure, limited only by the supercell size. As a consequence, the number of carbon atoms contained in each icosahedron, in principle, can be varied from zero to six. In our case, the number of carbon atoms in each icosahedron is varied from zero to two. To specify the symmetry of the RNG configuration, we assume an infinitely large $\mathrm{B}_{4} \mathrm{C}$ system. The probability that all polar sites are occupied by carbon atoms becomes equal since, in the RNG configuration, the icosahedral carbon atoms randomly substitute for any polar sites in the icosahedra. Its symmetry should eventually be similar to that of the $6 \mathrm{P}$ configuration, i.e., space group $R \overline{3} m$. This RNG configuration is the energetically most unfavorable among all of our disordered configurations.
The reason is that the possibility of having unfavorable carbon-carbon bonds in the structure is very high.

Figure 7 represents the Gibbs free energy under zeropressure conditions, plotted as a function of the temperature and with the free energy of the GS configuration taken as the zero value. The entropy $S$ and the free energy $G$ were calculated from the formula given in Sec. III. It should be noted that the ordered GS has zero entropy.

Even though the BD configuration has the lowest energy of the disordered configurations, the transition from the GS to the BD configuration is not predicted to happen. Instead we

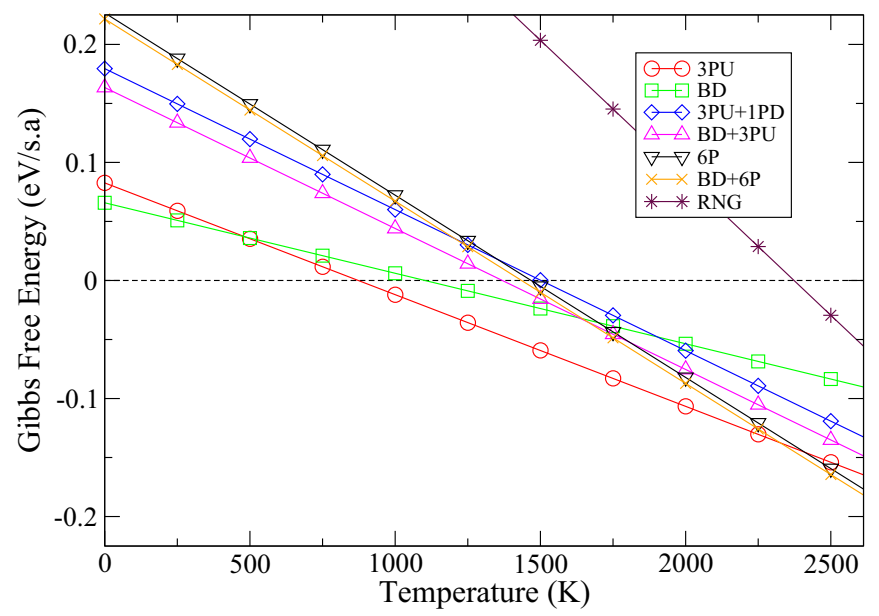

FIG. 7. (Color online) Difference in Gibbs free energy $(P=0)$ for seven disordered configurations, relative to the ground-state configuration (dashed line), as a function of temperature. 
observe a transition from the GS to the 3PU configuration at the temperature $870 \mathrm{~K}$. The reason is that the $3 \mathrm{PU}$ configuration, with three types of superatoms, has a larger configurational entropy than the BD configuration, with only two types. At this temperature, the icosahedral carbon atoms should gain enough energy and distribute themselves equally among the polar-up sites. Keeping in mind the findings for lattice symmetry above, we thus predict that there should be a transition of $\mathrm{B}_{4} \mathrm{C}$ from the GS configuration, where the monoclinic distortion exists, to rhombohedral symmetry $(R 3 m)$ at temperatures above $870 \mathrm{~K}$ under equilibrium conditions.

In our simulation, we observe a second configurational phase transition at $2325 \mathrm{~K}$ from the $3 \mathrm{PU}$ to the $\mathrm{BD}+6 \mathrm{P}$ configuration, where carbon atoms are distributed over all polar sites modeled with the Basis-2-type superatoms. At this transition temperature, a higher rhombohedral symmetry $(R \overline{3} m)$, in which the inversion symmetry is applied on average, is obtained. It is worth noting that as the second transition takes place at a very high temperature, which is close to the melting point of $\mathrm{B}_{4} \mathrm{C}(\sim 2650 \mathrm{~K})$, vibrational effects clearly cannot be neglected, i.e., the vibrational contributions of both the energy and the entropy should be taken into account to give a good description of the stability of phases at such a high temperature.

We do not observe any phase transition related to the type of disorder that involves four types of superatoms described by either Basis-1 or Basis-2.

As expected from the RNG configuration, the contribution of the configurational entropy is much higher than that of the other six configurations, as shown by its high slope in the Gibbs free energy diagram. The expression of the configurational entropy per superatom $S^{\mathrm{RNG}}$, obtained using Stirling's approximation, is given by

$$
S^{\mathrm{RNG}}=-6 k_{B}\left[\frac{1}{6} \ln \frac{1}{6}+\frac{5}{6} \ln \frac{5}{6}\right] .
$$

Even though the entropy of the RNG configuration is large, its free energy is still distinctly high and it is not even stable at high temperatures. This is because there are many unfavorable intericosahedral $\mathrm{C}-\mathrm{C}$ bonds existing in the supercell. We, consequently, disregard the possibility of havin it as the high-temperature phase.

Our findings are different from the predictions of Widom and Huhn $[31,49]$. Based on their study, the GS configuration is stable up to $600 \mathrm{~K}$ before it undergoes the configurational phase transition to the $6 \mathrm{P}$ configuration, where they claimed that carbon atoms became disordered among all six polar sites in the icosahedra, thus yielding the space group $R \overline{3} m$ on average. Their model were based on partition function creation with input from a limited number of individual configurations in small supercells. In such an analysis there exist inaccuracies in defect energetics due to defect-defect interactions and incomplete sampling of random-like configurations. On the other hand, our results reveal two configurational phase transitions, where the configuration with the full rhombohedral symmetry $(R \overline{3} m)$ is stable at much higher temperatures. Note that our calculations are based on the mean-field approximation, which is known to overestimate ordering transition temperatures by approximately a few hundred kelvins.
Based on our results we suggest an explanation for the experimental observation of rhombohedral symmetry $(R \overline{3} m)$ without monoclinic distortion even at low temperatures: The atomic diffusion in $\mathrm{B}_{4} \mathrm{C}$ is quenched above the critical ordering temperature, i.e., higher than the $870 \mathrm{~K}$ predicted as the first transition temperature, probably because of the very strong covalent bonds in the boron-carbon system, thus preventing the GS configuration with the monoclinic distortion from appearing. Experimentally, a crystalline boron carbide is normally obtained when synthesized or annealed from an initially amorphous phase at very high temperatures, where atomic diffusion is active, typically in the range between 1600 and $2000 \mathrm{~K}[32,50]$. In this range of temperatures, the crystalline phase formation temperature can possibly fall into either (1) the region where the $\mathrm{BD}+6 \mathrm{P}$ configuration is stable or (2) the region where the $3 \mathrm{PU}$ configuration is stable. In the first case, the full rhombohedral symmetry $(R \overline{3} \mathrm{~m})$ will obviously be achieved on average, corresponding to what has been reported in literature. However, in the second case, the predicted rhombohedral symmetry will be lacking inversion symmetry, thus being specified $R 3 m$ on average. The lack of inversion symmetry is in contrast to the reports of $R \overline{3} m$ symmetry in the experimental literature. One possibility is that real existing boron carbide, although possessing $R \overline{3} m$ symmetry globally, could display a short-range order tendency towards either predominantly polar-up or predominantly polar-down disorder, coexisting in different domains. In this case the global symmetry would be $R \overline{3} m$, with inversion symmetry present on average, while the energetics and the number of boronboron, carbon-carbon, and boron-carbon bonds of the system would still be better described by our 3PU configuration for polar disorder on one pole only. These questions require theoretical methods going beyond the mean-field description of configurational disorder presented here.

Also, future experimental work may shed light on the local configurations of polar carbon in $\mathrm{B}_{4} \mathrm{C}$. However, we note that the difference in atomic form factors for $\mathrm{x}$-ray diffraction between boron and carbon is very small and the lattice parameters, as listed in Table II, of the 3PU and the $\mathrm{BD}+6 \mathrm{P}$ configurations are practically identical. This similarity in $\mathrm{X}$-ray form factors, the almost-identical lattice parameters, and the fact that the difference between such $R \overline{3} m$ and $R 3 m$ configurations discussed above only involves partial occupation numbers of one of three carbon atoms in the structure should create considerable challenges to experimental determination of this aspect.

\section{Pressure effects}

Our approach makes it possible also to investigate the impact of high pressure on the configurational stability of the disordered $\mathrm{B}_{4} \mathrm{C}$ phases. In the present work, two configurations are considered, i.e. the GS and the 3PU configurations. As mentioned in Sec. III, the total energies at different fixed volumes of these two configurations are fitted to the third-order Birch-Murnaghan equation of state. The effects of pressure on the configurational stability are then determined by the Gibbs free energy given in Eq. (5). We have found that the applied pressure has a very small effect on the configurational stability of $\mathrm{B}_{4} \mathrm{C}$. The rate of change of the free energy is of the order 
of $10^{-5} \mathrm{eV} \mathrm{GPa}^{-1}$ atom $^{-1}$. In fact, this is because of the very high value of the bulk modulus of $\mathrm{B}_{4} \mathrm{C}$. The bulk moduli obtained for the GS and the 3PU configurations are 237.6 and $237 \mathrm{GPa}$, respectively, which are in good agreement with the experimental value, i.e., $247 \mathrm{GPa}$, given by Gieske et al. [51]. We also observed that the transition temperature of $\mathrm{B}_{4} \mathrm{C}$ from the GS to the 3PU configurations is raised with pressure at the rate of $6 \mathrm{~K}$ per $\mathrm{GPa}$. This is due to the slightly smaller volume of the GS configuration compared to the 3PU configuration.

Based on our calculations, applying a pressure of $15 \mathrm{GPa}$ would raise the transition temperature by approximately $90 \mathrm{~K}$. This increase in ordering temperature with pressure might be tried as a route to reach the predicted ordered GS configuration experimentally. However, recently Mikhaylushkin et al. [52] predicted that ordered $\mathrm{B}_{4} \mathrm{C}$ becomes unstable with respect to phase separation into diamond and pure boron at pressures above $20 \mathrm{GPa}$. This has the consequence that the use of even higher pressures in attempts to stabilize the (GS) monoclinic distorted phase in a temperature range where diffusion is active becomes limited by the appearance of phase separation.

However, it should be noted that Fujii et al.'s experiments [53] demonstrated that $\mathrm{B}_{4} \mathrm{C}$ is stable at pressures above $120 \mathrm{GPa}$, probably due to low diffusion at the low temperatures used, which made phase separation impossible.

\section{Electronic density of states}

In this subsection, the band gap and electronic DOS of the configurationally disordered $\mathrm{B}_{4} \mathrm{C}$ are discussed. Confirmed by experiments [54-57], boron carbide is a semiconducting material with a wide range of energy gaps, depending on its stoichiometry and quality. The indirect band gaps of $\mathrm{B}_{4} \mathrm{C}$ reported so far fall into the range between 0.48 and $2.5 \mathrm{eV}$ [54-57]. However, larger band gaps, within the range from 2.6 up to $3.0 \mathrm{eV}$, are obtained from LDA-based theoretical calculations $[10,15,48]$. This issue has, hence, been questionable because it is known from other materials that both LDA- and GGA-based electronic structure calculations typically underestimate, not overestimate, band gaps. Based on our calculations, a band gap as large as $3 \mathrm{eV}$, with the use of GGA-PBE for the exchange-correlation functional, is obtained for the GS configuration. A better accuracy for band-gap calculations is expected to be achieved by using the hybrid functional (HSE) for the exchange-correlation functional. It is known to resolve the problem of underestimating band gaps in standard LDA- and GGA-based calculations. The reason is that, within the HSE, the non-local Hartree-Fock exchange energy is included. Here we use this more reliable method to investigate the band gap of ideally ordered (GS) $\mathrm{B}_{4} \mathrm{C}$. As expected, our results show that the band gap of the GS configuration becomes even wider, $\sim 4 \mathrm{eV}$, when the HSE06 functional is used. However, calculating the band gaps from those disordered configurations constructed within very large supercells using the HSE06 is not doable due to the extremely expensive computational cost. Therefore, instead of using HSE06, we employ the MBJ exchange potential, which yields band gaps with an accuracy of about the same level as that obtained from the hybrid functional, but with much less computational effort. Within the MBJ exchange potential in combination with the GGA-PBE correlation, the band gap of the $\mathrm{GS} \mathrm{B}{ }_{4} \mathrm{C}$ is $\sim 3.7 \mathrm{eV}$. We thus prove, with these more reliable methods, that the huge difference between the computed and the experimentally measured band gaps for $\mathrm{B}_{4} \mathrm{C}$ does not arise from inaccuracies in the exchange-correlation functionals used. Consequently, we hypothesize that the shrinkage of the band gap in $\mathrm{B}_{4} \mathrm{C}$ originates from configurational disorder, either the equilibrium types discussed above or owing to defects caused by out-of-equilibrium synthesis. It is worth noting that, in some senses, the band-gap concept can be replaced by the mobility gap when one is dealing with disordered materials, because overlap between localized states in a band gap due to, e.g., different kinds of defects, and extended states from the band edges by, e.g., structural disorder, can take place, thus resulting in a smooth transition between these states. This is somewhat beyond our scope, since we do not investigate which states are dominated by defects or disordering effects. Consequently, only the band-gap term is used in the present work and the effects of configurational disorder on the band gap of $\mathrm{B}_{4} \mathrm{C}$ are discussed in detail in the paragraphs below.

We then investigate how different types of dilute defects affect the band gap of $\mathrm{B}_{4} \mathrm{C}$ based on the GGA-PBE functional. Except for the rotation of the icosahedral carbon atom at the polar sites, the other dilute defects created by substituting the carbon atom in the icosahedron, including distorted BDs, can reduce the band gap by about $0.15-0.3 \mathrm{eV}$. On the other hand, the defective chain configurations can shrink the band gap even more, down to (1) $1.6 \mathrm{eV}$ for $\left[\mathrm{B}_{12}+(\mathrm{C}-\mathrm{C}-\mathrm{C})\right]$ by creating midgap states $[48,58]$ and (2) $2.4 \mathrm{eV}$ for $\left[\mathrm{B}_{11} \mathrm{C}^{p(1)}+(\mathrm{C}-\mathrm{C}-\mathrm{B})\right]$ or $\left[\mathrm{B}_{11} \mathrm{C}^{p(1)}+(\mathrm{B}-\mathrm{C}-\mathrm{C})\right]$ by causing additional states near the conduction band edge.

The energy gaps of the disordered configurations, constructed using the SA-SQS technique, are listed in Table III and the electronic DOS for the GS, BD $+6 \mathrm{P}$, and RNG configurations are shown in Fig. 8. As clearly reported in the table, there is a very small change in the band gap $(\sim 0.1 \mathrm{eV})$ of the disordered configurations, induced by the random substitution of the icosahedral carbon atom among the polar sites on each icosahedron, i.e., the 3PU, 3PU + 1PD, and 6P configurations, whereas a reduction in the band gap by $\sim 0.3 \mathrm{eV}$ is found from those BD-based disordered configurations. These results are in accord with what is obtained from the dilute defect calculations. The band gap of the RNG configuration is considerably smaller compared to that of the GS configuration,

TABLE III. Electronic band gap of configurationally disordered $\mathrm{B}_{4} \mathrm{C}$. Abbreviations used are defined in the heading to Table II.

\begin{tabular}{lccc}
\hline \hline & \multicolumn{3}{c}{ Band gap (eV) } \\
\cline { 2 - 4 } Configuration & GGA-PBE & MBJ-GGA & HSE06 \\
\hline GS (ref.) & 3.00 & 3.72 & 4.13 \\
BD & 2.66 & 3.36 & - \\
3PU & 2.98 & 3.72 & - \\
3PU + 1PD & 2.87 & 3.60 & - \\
6P & 2.91 & 3.69 & - \\
BD + 3PU & 2.63 & 3.31 & - \\
BD + 6P & 2.72 & 3.40 & - \\
RNG & 2.01 & 2.76 & - \\
\hline \hline
\end{tabular}




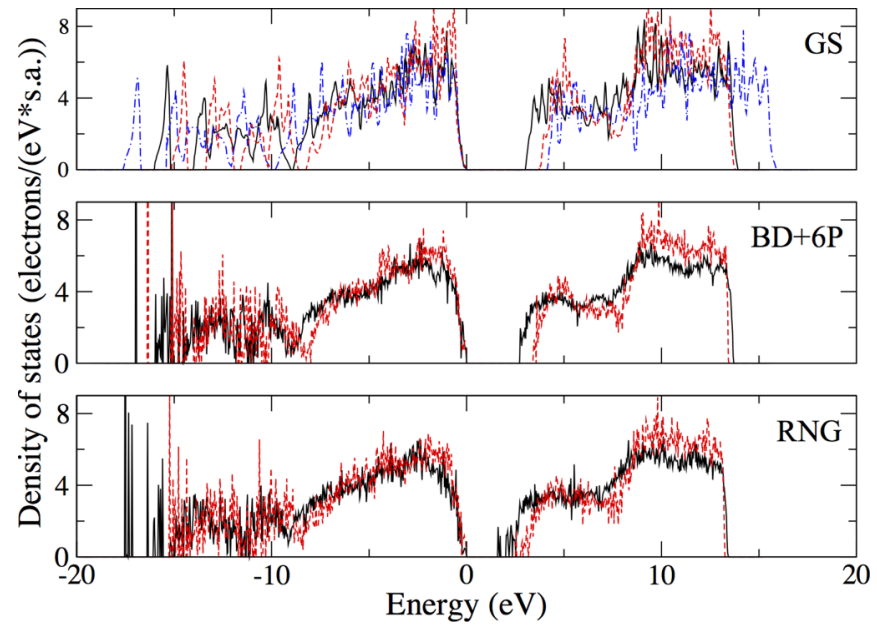

FIG. 8. (Color online) Density of states (DOS) of ordered/disordered $\mathrm{B}_{4} \mathrm{C}$. GS: Solid black line, dashed (red) line, and dashed-dotted (blue) line indicate the electronic DOS of the (GS) configuration, obtained using the GGA-PBE, MBJ-GGA, and HSE06, respectively, for the exchange-correlation functional. $\mathrm{BD}+6 \mathrm{P}$ and RNG: Electronic DOS of the (BD+6P) and (RNG) configurations, respectively, with the GGA-PBB (solid black line) and MBJ-GGA [dashed (red) line] exchange-correlation functionals.

2.01 (2.76) eV for the GGA-PBE (MBJ-GGA) functional, due to extra electronic states adjacent to the conduction band edge (see Fig. 8). There are also midgap states in the RNG configuration, at 1.6 and $2.5 \mathrm{eV}$ above the valence-band edge for the GGA-PBE and MBJ-GGA functionals, respectively. These additional states seem to be similar to the cases of a defective chain, i.e., (C-C-C), (B-C-C), and (C-C-B) chains, as mentioned above. We know from previous works $[48,58]$ that the replacement of the central boron atom in the chain by a carbon atom results in midgap states. However, in our case, we did not introduce any chain defect into the configuration. We, therefore, suggest that the additional states, both near the conduction band edge and in the middle of the band gap, originate from the high concentration of $\mathrm{C}-\mathrm{C}$ bonds existing in the RNG structure. The DOS of the GS, BD + 6P, and RNG configurations are illustrated in Fig. 8. Note that, apart from the size of the band gap, the DOS for the $\mathrm{BD}, \mathrm{BD}+3 \mathrm{PU}$, $3 \mathrm{PU}, 3 \mathrm{PU}+1 \mathrm{PD}$, and $6 \mathrm{P}$ configurations are more or less the same (on average) as that of the GS configuration. Even though our results reveal a trend of band-gap decrease with increasing degree of configurational disorder, they do not explain the experimental results quantitatively, as one would have expected a smaller band gap for GGA calculations in that case. Without taking into account these midgap states, the RNG configuration, which has the smallest band gap-even below (close to) the largest experimental value reported when the GGA-PBE (MBJ-GGA) functional is used-has a very high value of the free energy, even at high temperatures. While we do not expect such a state to appear in equilibrium, the smaller band gap indicates that the presence of high-energy defects caused by difficulties in reaching equilibrium during synthesis might be the cause for the lower than expected experimental band gap. The large spread in the experimental reports of the band gap is in itself an indication of the sensitivity of the electronic properties of $\mathrm{B}_{4} \mathrm{C}$ to structural disorder. This is in accord with the study by Ivashchenko et al. [48], in which they investigated the electronic DOS of amorphous $\mathrm{B}_{4} \mathrm{C}$ using molecular dynamic simulations. Their results reveal a DOS minimum, instead of a band gap, in the amorphous $\mathrm{B}_{4} \mathrm{C}$, yielding semimetallic properties.

\section{CONCLUSIONS}

We have studied configurational disorder in $\mathrm{B}_{4} \mathrm{C}$ using first-principles calculations. A method to access different disordered configurations, namely, the superatom-special quasirandom structure technique, is demonstrated. The method is a combination of a concept of superatoms to describe low-energy defects in a complex structure and a distribution of the superatoms like a random alloy using the SQS technique. Using this SA-SQS technique, the properties of the configurationally disordered $\mathrm{B}_{4} \mathrm{C}$, e.g., symmetry and electronic DOS, become accessible. We predict two configurational phase transitions (at $P=0 \mathrm{GPa}$ ), at $\sim 870$ and $\sim 2325 \mathrm{~K}$, respectively, from the ordered to the increasingly disordered phases, within the mean-field approximation. After undergoing the first transition, the higher rhombohedral symmetry without the inversion $(R 3 m)$ in $\mathrm{B}_{4} \mathrm{C}$ is restored, due to disorder of icosahedral carbon among the three polar-up sites in the structure, meanwhile the full rhombohedral symmetry $(R \overline{3} m)$ reported in experiments is restored after the second transition, where the random substitution of the icosahedral carbon atoms at all six polar sites and the BDs exist in the icosahedral structure of $\mathrm{B}_{4} \mathrm{C}$ at high concentrations. Details on the icosahedral carbon disorder and the difference in the $R 3 m$ and $R \overline{3} m$ states deserve further attention using methods going again beyond the meanfield approximation for configurational thermodynamics and, most importantly, including vibrational effects.

Nevertheless, our present level of calculations provides an explanation for why the $\mathrm{B}_{4} \mathrm{C}$ ordered GS with monoclinic distortion has so far not been observed: The atomic diffusion in $\mathrm{B}_{4} \mathrm{C}$ is likely to be quenched above the critical ordering temperature, prohibiting access to the low-temperature ordered state. By investigating the impact of high pressure on the configurational stability of the disordered $\mathrm{B}_{4} \mathrm{C}$ phases, we have found that the configurationally ordering/disordering transition temperature increases with the pressure exerted on $\mathrm{B}_{4} \mathrm{C}$. Also, by considering the electronic DOS, we have found that the band gap of $\mathrm{B}_{4} \mathrm{C}$ is sensitive to the degree of disorder, also for configurational disorder in the icosahedra. Such changes in the electronic structure demonstrate that, apart from the stoichiometry, also the configurations of both the chain and the icosahedra influence the electronic structure and the band gap of $\mathrm{B}_{4} \mathrm{C}$.

\section{ACKNOWLEDGMENTS}

Carina Höglund, Mewlude Imam, and Henrik Pedersen are acknowledged for useful discussions. Financial support by the Swedish Research Council (VR) through Young Researcher Grant No. 621-2011-4417 is gratefully acknowledged by B.A. The support from Swedish Research Council (VR) Project No. 2011-42-59, LiLi-NFM, and the Swedish Government Strategic Research Area Grant in Materials Science to the 
AFM research environment at LiU are acknowledged by S.I.S. Financial support from the KAW project Isotopic Control for Ultimate Materials Properties is greatly appreciated by J.B.
Simulations were carried out using supercomputer resources provided by the Swedish National Infrastructure for Computing (SNIC) at the National Supercomputer Center (NSC).
[1] J. L. Lacy, A. A. thanasiades, L. Sun, C. S. Martin, T. D. Lyons, M. A. Foss, and H. B. Haygood, Nucl. Instrum. Methods Phys. Res. A 652, 359 (2011).

[2] C. Höglund, J. Birch, K. Andersen, T. Bigault, J.-C. Buffet, J. Correa, P. van Esch, B. Guerard, R. Hall-Wilton, J. Jensen, A. Khaplanov, F. Piscitelli, C. Vettier, W. Vollenberg, and L. Hultman, J. Appl. Phys. 111, 104908 (2012).

[3] O. Knotek, E. Lungscheider, and C. W. Siry, Surf. Coat. Technol. 91, 167 (1997).

[4] D. Simeone, C. Mallet, P. Dubuisson, G. Baldinozzi, C. Gervais, and J. Maquet, J. Nucl. Mater. 277, 1 (2000).

[5] M. Carrard, D. Emin, and L. Zuppiroli, Phys. Rev. B 51, 11270 (1995).

[6] D. Emin, J. Solid State Chem. 179, 2791 (2006).

[7] J. E. Zorzi, C. A. Perottoni, and J. A. H. da Jornada, Mater. Lett. 59, 2932 (2005).

[8] S. Adenwalla, P. Welsch, A. Harken, J. I. Brand, A. Sezer, and B. W. Robertson, Appl. Phys. Lett. 79, 4357 (2001).

[9] H. Werheit, Mater. Sci. Eng. B 29, 228 (1995).

[10] D. M. Bylander, L. Kleinman, and S. Lee, Phys. Rev. B 42, 1394 (1990).

[11] D. M. Bylander and L. Kleinman, Phys. Rev. B 43, 1487 (1991).

[12] R. Lazzari, N. Vast, J. M. Besson, S. Baroni, and A. Dal Corso, Phys. Rev. Lett. 83, 3230 (1999).

[13] F. Mauri, N. Vast, and C. J. Pickard, Phys. Rev. Lett. 87, 085506 (2001).

[14] N. Vast, J. Sjakste, and E. Betranhandy, J. Phys.: Conf. Ser. 176, 012002 (2009).

[15] K. Shirai, J. Superhard Mater. 32, 205 (2010).

[16] K. Shirai, J. Superhard Mater. 32, 336 (2010).

[17] V. Domnich, S. Reynaud, R. A. Haber, and M. Chhowalla, J. Am. Ceram. Soc. 94, 3605 (2011).

[18] D. E. Taylor, J. W. McCauley, and T. W. Wright, J. Phys.: Condens. Matter 24, 505402 (2012).

[19] H. K. Clark and J. L. Hoard, J. Am. Chem. Soc. 65, 2115 (1943).

[20] H. L. Yakel, Acta Crystallogr. Sec. B 31, 1797 (1975).

[21] B. Morosin, A. W. Mullendore, D. Emin, and G. A. Slack, AIP Conf. Proc. 140, 70 (1986).

[22] B. Morosin, G. H. Kwei, A. C. Lawson, T. L. Aselage, and D. Emin, J. Alloys Compd. 226, 121 (1995).

[23] B. Morosin, T. Aselage, and R. Feigelson, MRS Proc. 97, 145 (1987).

[24] A. C. Larson, AIP Conf. Proc. 140, 109 (1986).

[25] U. Kuhlmann and H. Werheit, J. Alloys Compd. 189, 249 (1992).

[26] G. H. Kwei and B. Morosin, J. Phys. Chem. 100, 8031 (1996).

[27] R. Schmechel and H. Werheit, J. Phys.: Condens. Matter 11, 6803 (1999).

[28] J. E. Saal, S. Shang, and Z. K. Liu, Appl. Phys. Lett. 91, 231915 (2007).

[29] M. M. Balakrishnarajan, P. D. Pancharatna, and R. Hoffmann, New J. Chem. 31, 473 (2007).
[30] R. Raucoules, N. Vast, E. Betranhandy, and J. Sjakste, Phys. Rev. B 84, 014112 (2011).

[31] M. Widom and W. P. Huhn, Solid State Sci. 14, 1648 (2012).

[32] C. Pallier, J.-M. Leyssale, L. A. Truflandier, A. T. Bui, P. Weisbecker, C. Gervais, H. E. Fischer, F. Sirotti, F. Teyssandier, and G. Chollon, Chem. Mater. 25, 2618 (2013).

[33] A. Zunger, S.-H. Wei, L. G. Ferreira, and J. E. Bernard, Phys. Rev. Lett. 65, 353 (1990).

[34] P. E. Blöchl, Phys. Rev. B 50, 17953 (1994).

[35] G. Kresse and J. Hafner, Phys. Rev. B 48, 13115 (1993).

[36] J. Perdew, K. Burke, and M. Ernzerhof, Phys. Rev. Lett. 77, 3865 (1996).

[37] H. J. Monkhorst and J. D. Pack, Phys. Rev. B 13, 5188 (1976).

[38] P. E. Blöchl, O. Jepsen, and O. K. Andersen, Phys. Rev. B 49, 16223 (1994).

[39] A. D. Becke and E. R. Johnson, J. Chem. Phys. 124, 221101 (2006).

[40] F. Tran and P. Blaha, Phys. Rev. Lett. 102, 226401 (2009).

[41] J. Paier, M. Marsman, K. Hummer, G. Kresse, I. C. Gerber, and J. G. Ángyán, J. Chem. Phys. 124, 154709 (2006).

[42] A. V. Krukau, O. A. Vydrov, A. F. Izmaylov, and G. E. Scuseria, J. Chem. Phys. 125, 224106 (2006).

[43] K. Momma and F. Izumi, J. Appl. Crystallogr. 44, 1272 (2011).

[44] J. M. Sanchez, F. Ducastelle, and D. Gratias, Physica A 128, 334 (1984).

[45] F. D. Murnaghan, Proc. Natl. Acad. Sci. USA 30, 382 (1944).

[46] F. Birch, Phys. Rev. 71, 809 (1947).

[47] See Supplemental Material at http://link.aps.org/supplemental/ 10.1103/PhysRevB.90.024204 for the SA-SQS structure files of the fully relaxed 3PU-, 6P-, and (BD + 6P)-disordered configurations.

[48] V. I. Ivashchenko and V. I. Shevchenko, Phys. Rev. B 80, 235208 (2009).

[49] W. P. Huhn and M. Widom, J. Stat. Phys. 150, 432 (2013).

[50] J. K. Sonber, T. S. R. Ch. Murthy, C. Subramanian, R. K. Fotedar, R. C. Hubli, and A. K. Suri, Trans. Indian Ceram. Soc. 72, 100 (2013).

[51] J. H. Gieske, T. L. Aselage, and D. Emin, AIP Conf. Proc. 231, 376 (1991)

[52] A. S. Mikhaylushkin, X. Zhang, and A. Zunger, Phys. Rev. B 87, 094103 (2013).

[53] T. Fujii, Y. Mori, H. Hyodo, and K. Kimura, J. Phys.: Conf. Ser. 215, 012011 (2010).

[54] H. Werheit, H. Binnenbruck, and A. Hausen, Physica Status Solidi B 47, 153 (1971).

[55] H. Werheit, M. Laux, U. Kuhlmann, and R. Telle, Physica Status Solidi B 172, K81 (1991).

[56] H. Werheit, C. Janowitz, R. Schmechel, T. Tanaka, and Y. Ishizawa, J. Solid State Chem. 133, 132 (1997).

[57] H. Werheit, J. Phys.: Condens. Matter 18, 10655 (2006).

[58] H. Dekura, K. Shirai, and A. Yanase, J. Phys.: Conf. Ser. 215, 012117 (2010). 